\title{
Domination Invariant of a Diameter Constrained Network Reliability Model
}

\author{
Héctor Cancela ${ }^{\mathrm{a}, 1}$ and Louis Petingi ${ }^{\mathrm{b}, 2}$ \\ ${ }^{a}$ Departamento de Investigación Operativa, Instituto de Computación, \\ Facultad de Ingeniería, Universidad de la República \\ J. Herrera y Reissig 565, Montevideo, Uruguay \\ ${ }^{\mathrm{b}}$ Computer Science Department, College of Staten Island, \\ City University of New York
}

2800 Victory Boulevard, 1N, Staten Island, N. Y. 10314, USA

\begin{abstract}
Let $G=(V, E)$ be a digraph with a distinguished set of terminal vertices $K \subseteq V$ and a vertex $s \in K$. We define the $s, K$-diameter of $G$ as the maximum distance between $s$ and any of vertices of $K$. If the arcs fail randomly and independently with known probabilities (vertices are always operational), the Diameter-constrained $s, K$-terminal reliability of $G, R_{s, K}(G, D)$, is defined as the probability that surviving arcs span a subgraph whose $s, K$-diameter does not exceed $D$.

The Diameter-constrained network reliability is a special case of coherent system models, where the domination invariant has played an important role, both theoretically and for developing algorithms for reliability computation. In this work, we completely characterize the domination of diameter-constrained network models, giving a simple rule for computing its value: if the digraph either has an irrelevant edge, includes a dicycle or includes a dipath from $s$ to a node in $K$ longer than $D$, its domination is 0 ; otherwise, its domination is -1 to the power $|E|-|V|+1$.
\end{abstract}

Key words: Graph theory, domination, diameter-constrained network reliability.

\section{Introduction and reliability model}

A communication network can be modeled by a digraph (directed graph) $G=(V, E)$ where $V$ and $E$ are the set of vertices and arcs respectively of $G$.

$\overline{1}$ cancela@fing.edu.uy

2 petingi@postbox.csi.cuny.edu 
Moreover the failures of the network components can be represented by assigning probabilities of failure to the vertices and/or arcs of its underlying digraph. A widely used probabilistic model is the one where the arcs fail randomly and independently with known probabilities, and where the vertices are always operational; from this point on, when we mention a probabilistic digraph, we will refer to this model.

Let $G=(V, E)$ be a probabilistic digraph, with terminal vertex set $K \subseteq V$, vertex $s \in K$, and distance bound $D$; the $s, K$-diameter of $G$ is the maximum distance from $s$ to any vertex $u \in K$. The Diameter-constrained $s, K$-terminal reliability $R_{s, K}(G, D)$ is defined (7) as the probability that the surviving arcs span a subgraph of $G$ whose $s, K$-diameter does not exceed $D$, or equivalently, as the probability that for each vertex $u \in K$, there exists an operating $s, u$ dipath from $s$ to $u$ of at most $D$ arcs. This reliability measure subsumes the classical Source-to- $K$-terminal reliability $R_{s, K}(G)$ of a probabilistic digraph $G$, which is the probability that the surviving arcs span a subgraph where there exists an operational $s, u$-dipath between $s$ and $u, u \in K$ : noting that the longest $s, u$-dipath in $G$ has at most $n-1$ arcs, where $n$ is the number of nodes of $G$, we have that $R_{s, K}(G)$ is equal to $R_{s, K}(G, D)$ for $D=n-1$.

We introduce here additional definitions and notation:

(i). Let $G=(V, E, \mathbf{P}(E))$ be a probabilistic digraph with a distinguished set $K \subseteq V$, vertex $s \in K$, and $D \in Z^{+}$, with $1 \leq D \leq n-1$, where $n=|V|$, and where $\mathbf{P}: E \mapsto[0,1]$ are the operational probabilities of the arcs in set $E$. We represent the operational probability of an arc (or arc reliability) $x \in E$ as $p(x)=1-q(x)(q(x)$ is the probability of failure).

(ii). Let the sample space $\Omega$ represent the set of all possible subsets of $E$, corresponding to sets of operational arcs (i.e. $\Omega=2^{E}$ ).

(iii). Under the assumption of independent arc failures, each $H \in \Omega$ has occurrence probability

$$
P(H)=\prod_{x \in H} p(x) \prod_{x \notin H} q(x) .
$$

(iv). $H \in \Omega$ is a pathset or operating state if $H$ spans a subgraph whose $s, K$ diameter is at most $D$.

(v). Let $\mathbf{O}_{K}^{D}(E)=\{H \in \Omega: H$ is a pathset $\}$.

(vi). An operating state $H$ of $\mathbf{O}_{K}^{D}(E)$ is called a minpath if $H-\left\{x_{i}\right\} \notin \mathbf{O}_{K}^{D}(E)$, for all $x_{i} \in H$ (i.e, a minpath is a minimal operating state).

(vii). A $K$-tree $T$ of a digraph $G$ is a tree, rooted at $s$, covering all the vertices of $K$, and such that any pendant vertex $u$ of $T$ belongs to $K$. In addition, a $K$-tree whose $s, K$-diameter is at most $D$ is called a $D, K$-tree.

(viii). $G$ is called a $D, K$-digraph, if every arc of $G$ lies in some $D, K$-tree. Taking into account definition (vii), we know that if $G$ is a $D, K$-digraph, then node $s$ has indegree 0 ; and every arc of $G$ belongs to a path from $s$ to some node in $K$; we say that $G$ is rooted at $s$. 
The following lemma gives a characterization of the minpaths $M$ of $\mathbf{O}_{K}^{D}(E)$ :

Lemma 1 For a digraph $G=(V, E)$, terminal set $K$, vertex $s \in K$, and bound $D$, then $M$ is a minpath of $G$ if and only if it is a $D, K$-tree.

From the definition of $R_{s, K}(G, D)$ and definition (v) one gets

$$
R_{K}(G, D)=\operatorname{Pr}\left(\mathbf{O}_{K}^{D}(E)\right)=\sum_{H \in \mathbf{O}_{K}^{D}(E)} \prod_{e \in H} p(e) \prod_{e \notin H} q(e) .
$$

We next discuss the definition of the domination invariant in the case of general coherent systems, and in the case of diameter-constrained network reliability.

\section{Domination}

A graph invariant called the reliability domination of a graph $G$ was introduced by Satyanarayana and Prabhakar (8) for the classical network reliability models, and has since been explored by several researchers in reliability theory $(1 ; 2 ; 3 ; 5 ; 6)$. The reliability domination plays an important role, allowing to efficiently implement the principle of Inclusion-Exclusion of probability theory applied to the evaluation of reliability measures for general reliability systems.

Let $E$ be a finite set, and $P(E)$ be the power set of $E$. A nonempty subset $\mathbf{C} \subseteq P(E)$ is called a clutter of $E$ if for any two elements $C_{1}, C_{2} \in \mathbf{C}$, whenever $C_{1} \subseteq C_{2}$, then $C_{1}=C_{2}$. A pair $(E, \mathbf{C})$ will be referred to as a system and a system is coherent if each element of $E$ is contained in some element of $\mathbf{C}$. A formation of $(E, \mathbf{C})$ is a collection of elements of $\mathbf{C}$ whose union yield $E$. The signed domination of the system $(E, \mathbf{C})$, denoted $d(E, \mathbf{C})$, is defined as the number of odd formations minus the number of even formations of $E$, where a formation is said to be odd or even if it is of odd or even cardinality respectively. Trivially by the previous definitions, a non-coherent system has no formations, so its signed domination is 0 .

The clutters associated with the operation and failure of a specific element $x \in E$ are defined as follows. Let $\mathbf{C}-x=\{C-x: C \in \mathbf{C}\}$ and $\mathbf{C}_{-x}=\{C \in$ $\mathbf{C}: x \notin C\}$. Now $\mathbf{C}_{-x}$ is clearly a clutter but $\mathbf{C}-x$ may not be one. We define $\mathbf{C}_{+x}$ to be the collection of elements of $\mathbf{C}-x$ which are not proper supersets of some element of $\mathbf{C}-x$. For an element $x \in E, \mathbf{C}_{-x}$ and $\mathbf{C}_{+x}$ are called the minors with respect to $x$ of $\mathbf{C}$. Huseby $(5 ; 6)$ showed the following result:

Theorem 1 If $(E, \mathbf{C})$ is a system, with $x \in E$, and minors $\mathbf{C}_{-x}$ and $\mathbf{C}_{+x}$ of $\mathbf{C}$, then $d(E, \mathbf{C})=d\left(E-\{x\}, \mathbf{C}_{+x}\right)-d\left(E-\{x\}, \mathbf{C}_{-x}\right)$. 
We look now at the case of the diameter-constrained $s, K$ terminal reliability of a digraph $G=(V, E)$ with $K \subseteq V, s \in K$, and diameter bound $D$. The system underlying our model is $\left(E, \mathbf{F}_{D, K}(G)\right)$, where $E$ is the set of $\operatorname{arcs}$ of $G$, and where $\mathbf{F}_{D, K}(G)$ is the collection of $D, K$-trees of $G$. A formation $F$ of $G$ is then a collection of $D, K$-trees of $G$ whose union is $E$, the set of $\operatorname{arcs}$ of $G$. The signed domination of a digraph $G=(V, E)$, denoted $d\left(E, \mathbf{F}_{D, K}(G)\right)$, with respect to a given subset $K \subseteq V, s \in K$, and bound $D$, is the number of odd minus the number of even formations of $G$.

For brevity, in what follows we will use the standard notation $\mathbf{C}$ to represent $\mathbf{F}_{D, K}(G)$, which is the clutter set in the diameter-constrained model. Also we denote the domination $d\left(E, \mathbf{F}_{D, K}(G)\right)$ as $d_{D, K}(G)$. In addition, we observe that if $x$ is an arc of $G$, then $T$ is a $D, K$-tree of $G$ such that $x \notin T$ iff $T$ is a $D, K$-tree of $G-x$. Therefore $d\left(E-\{x\}, \mathbf{C}_{-x}\right)=d_{D, K}(G-x)$. Using this notation, the equation in Theorem 1 can be re-written as

$$
\begin{aligned}
d_{D, K}(G) & =d\left(E-\{x\}, \mathbf{C}_{+x}\right)-d\left(E-\{x\}, \mathbf{C}_{-x}\right) \\
& =d\left(E-\{x\}, \mathbf{C}_{+x}\right)-d_{D, K}(G-x),
\end{aligned}
$$

We next state the main results of this work, which are a characterization of the domination for diameter-constrained reliability models, and we discuss how these results can be used to compute the reliability of a network.

\section{Characterization of the domination and application to reliability evaluation}

Let $G=(V, E)$ be a digraph with terminal set $K, e=|E| \operatorname{arcs,} n=|V|$ vertices, and let $D$ be the diameter bound. We define the following operation:

- $\mathbf{L P}(G, s, K)$. If $G$ is $s, K$ connected, this operation returns the length of the longest dipath from $s$ to any vertex $u \in K$; otherwise it returns $\infty$.

The computation of $\mathbf{L P}(G, s, K)$ is in the $N P$-complete class; but it is of polynomial complexity if $G$ is an acyclic digraph.

We observe that if $G$ is not a $D, K$ digraph, there are some arcs in $E$ which are not covered by any $D, K$ tree; so that the corresponding system is non coherent, and there are no formations over the clutter $\mathbf{F}_{D, K}(G)$ able to cover $E$. As a result, the domination is zero. Consequently, from now on, we will restrict ourselves to the case of $D, K$ digraphs. For these digraphs, the domination is completely characterized by the following theorems: 
Theorem 2 Let $G=(V, E)$ be a cyclic $D, K$ digraph with terminal set $K$, $n=|V|$ vertices, $n>2$, and let $D$ be the diameter bound. Then $d_{D, K}(G)=0$.

Theorem 3 Let $G=(V, E)$ be a acyclic $D, K$ digraph with terminal set $K$, $e=|E|$ arcs, $n=|V|$ vertices, and let $D$ be the diameter bound, then

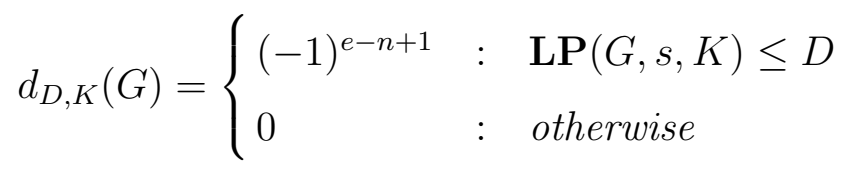

Both theorems can be proved by complete induction over the number of edges of the digraph, and using two different reductions, which preserve the existence of dicycles and the relation of the length of the longest dipath and the diameter bound. One reduction is applied when all the nodes adjacent to $s$ have indegree 1 ; in this case, we can "contract" all the arcs leaving $s$, and obtain a new digraph $G^{*}$ with diameter bound $D-1$ and with the same domination value as $G$. The other reduction is used when there is at least one node adjacent to $s$ with indegree greater than 2 ; if $x=(s, u)$, then it can be proved that $d\left(E-\{x\}, \mathbf{C}_{+x}\right)=0$, and by Equation 3 we have $d_{D, K}(G)=-d_{D, K}(G-x)$.

We give a short proof outline:

- Lemma: if for all $u$ adjacent to $s$, indegree $(\mathrm{u})=1$; then it is possible to contract all the arcs from $s$, creating a new graph $G^{*}$. We define a corresponding new terminal set $K^{*}$ with the following properties:

- if $G$ is a $D, K$ graph, $G^{*}$ is a $D-1, K^{*}$ graph

- $G^{*}$ is cyclic iff $G^{*}$ is cyclic.

- $L P\left(G^{*}\right)=L P(G)-1$

- $d_{D-1, K^{*}}\left(G^{*}\right)=d_{D, K}(G)$

- Theorem: Suppose $G$ is $D, K$ graph (else, dom=0).

Induction hypothesis: theorems true for $|E| \leq m$.

Thesis: true for $|E|=m+1$.

Proof:

Case a) if for all $u$ adjacent to $s$, indegree $(u)=1$; then by lemma trivial.

Case a) if there exists $u$ adjacent to $s$, indegree $(u)>1$. Then consider $x=(s, u)$.

Prove that $d\left(E-\{x\}, \mathbf{C}_{+x}\right)=0$ (by looking at other edges leading to $u$, which are irrelevant in the new clutter).

By Equation 3 we have $d_{D, K}(G)=-d_{D, K}(G-x)$.

See that $G-x$ is cyclic iff $G$ is cyclic; and that $L P(G-x)=L P(G)$. Using then the induction hypothesis, the thesis follows.

When we take $D=n-1$, we obtain the classical Source-to- $K$-terminal reliability model as a particular case. As all dipaths are of length smaller than 
$n-1$, then trivially $\mathbf{L P}(G, s, K) \leq D$ unless the graph is not $s, K$ connected. In this case, the characterization reduces to the results in (8), i.e. that the domination is 0 if there is a dicycle, and $(-1)^{e-n+1}$ otherwise.

These results are useful for computing the reliability of a given network. For a digraph $G=(V, E)$, terminal set $K$, and vertex $s \in K$, let $\mathbf{M}=$ $\left\{M_{1}, M_{2}, \ldots, M_{l}\right\}$ be the set of minpaths of $\mathbf{O}_{K}^{D}(E)$. Define $E_{i}$ to be the event that all the arcs of $M_{i}$ operate. By Inclusion-Exclusion we obtain

$$
\begin{aligned}
R_{s, K}(G, D)= & \operatorname{Pr}\left(\bigcup_{i=1}^{l} E_{i}\right)=\sum_{i} \operatorname{Pr}\left(E_{i}\right)-\sum_{i<j} \operatorname{Pr}\left(E_{i} E_{j}\right)+\ldots \\
& +(-1)^{l+1} \operatorname{Pr}\left(E_{1} E_{2} \ldots E_{l}\right)
\end{aligned}
$$

where the event $E_{i} E_{j} \ldots E_{m}$ is the event that all the arcs of the subgraph obtained by the union of $M_{i}, M_{j}, \ldots, M_{m}$ are operating.

In Equation (4), the terms correspond to subgraphs obtained by the union of minpaths. As discussed previously, for the Diameter-constrained $K$-terminal reliability of a digraph $G$, with terminal set $K$, vertex $s \in K$, and diameter bound $D$, the minpaths are $D, K$-trees, the formations are sets of minpaths, and the subgraphs are $D, K$-digraphs. The same $D, K$-digraph can be obtained from different formations; this means that it may appear in Equation (4) more than once, sometimes with positive sign, and sometimes with negative sign, depending if the corresponding formation has an odd or or an even number of $D, K$-trees. In fact, its net contribution will be exactly the number of odd minus the number of even formations of the graph, i.e., its domination invariant. Thus using these facts and the above definitions, we can rewrite Equation (4) as

$$
R_{s, K}(G, D)=\sum_{H \in \mathbf{H}} d_{D, K}(H) \operatorname{Pr}(H)
$$

where $\mathbf{H}$ is the class of all $D, K$-digraphs of $G$, and $\operatorname{Pr}(H)$ is the probability that the arcs of $H$ are operative.

\section{Acknowledgment}

This work was funded by Grant \#64407-00-33 of the City University of New York Research Foundation. The participation of Héctor Cancela was also supported by project PAIR of the INRIA, France. 


\section{References}

[1] Agrawal A., Barlow R.E.: A survey of network reliability and domination theory. Operations Research 32 (1984) 478-492.

[2] Barlow R.E.: Set-theoretic signed domination of coherent systems. Technical Report ORC 82-1, University of California, Berkeley (1982).

[3] Barlow R.E., Iyer S.: Computational complexity of coherent systems and the reliability polynomial, Probability in the Engineering and Information Sciences 2 (1988) 461-469.

[4] Colbourn, C.: The Combinatorics of Network Reliability, Oxford University Press (1987).

[5] Huseby, A.B.: A unified theory of domination and signed with applications to exact reliability domination, Statistical Research Report, Institute of Mathematics, University of Oslo, Norway (1984).

[6] Huseby, A.B.: Domination Theory and the crapo- $\beta$-invariant, Networks 19 (1989) 135-149.

[7] Petingi L., Rodriguez J.: Reliability of Networks with Delay Constraints. Congressus Numerantium 152 (2001) 117-123.

[8] Satyanarayana, A., Prabhakar A.: New topological formula and rapid algorithm for reliability analysis of complex networks, IEEE Transactions on Reliability R-27-2 (1978) 82-97. 